\title{
Classification of Cryptocurrency: A Review of the Literature
}

\section{Wan Nur Izzah Wan Muhamad Fokria*, Engku Muhammad Tajuddin Engku Ali ${ }^{\text {b }}$, Nadhirah Nordin $^{\mathrm{c}}$, Wan Mohd Yusof Wan Chik ${ }^{\mathrm{d}}$, Sumayyah Abdul Aziz ${ }^{\mathrm{e}}$, Ahmad Jazlan Mat Jusoh ${ }^{\mathrm{f}}$}

\author{
a*Universiti Sultan Zainal Abidin, Kuala Nerus, Terengganu, Malaysia. E-mail: izzahfokri.we@ gmail.com

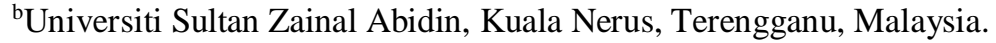 \\ ${ }^{c}$ Universiti Sultan Zainal Abidin, Kuala Nerus, Terengganu, Malaysia. \\ ${ }^{\mathrm{d} U n i v e r s i t i ~ S u l t a n ~ Z a i n a l ~ A b i d i n, ~ K u a l a ~ N e r u s, ~ T e r e n g g a n u, ~ M a l a y s i a . ~}$ \\ eUniversiti Sultan Zainal Abidin, Kuala Nerus, Terengganu, Malaysia. \\ fUniversiti Sultan Zainal Abidin, Kuala Nerus, Terengganu, Malaysia.
}

Article History: Received: 11 January 2021; Accepted: 27 February 2021; Published online: 5 April 2021

\begin{abstract}
Technological development has affected the global financial industry. The use of digital currency is increasingly gaining a place among the world's population, so much so that there are 2486 types of digital currency on record. Scholars in Islamic finance as well as Fatwa institutions all over the world have delivered their religious decree concerning the digital currency; hence, most discussions about the use of the decree was only directed at Bitcoin as a medium of payment although some digital currencies have other functions, such as being utility and security tokens. Therefore, the decree concerning other digital currencies cannot apply the decree issued for Bitcoin only because each digital currency has a different conceptual framework. Hence, this study, which applied the qualitative approach and a descriptive research design, intended to analyse the classification of digital currencies according to their function and characteristics. The findings show that digital currency is classified into coins, currency, tokens, payment tokens, utility tokens and security or asset tokens. Coins function as a medium of payment and store of value that was developed using its own blockchain. Currency is a medium of exchange and can be exchanged with any form of money, including the crediting or debiting of an account. Tokens represent services, financial instruments or infrastructure that is developed using the blockchain technology of other digital currencies. Tokens are divided into three types according to their function, namely payment tokens, utility tokens and security or asset tokens. The classification of digital currencies provides a guideline for the public who wish to carry out transactions using digital currencies. It is important to ensure that transactions carried out in accordance with the syarak will help alleviate cases of fraud related to investment and sale of digital currencies.
\end{abstract}

Keywords: Classification, Digital Currency, Coin, Token, Bitcoin.

\section{Introduction}

Islam welcomes technological development that produces innumerable innovations in various sectors around the world, including the financial sector, if it is Syariah compliant. Each country has an authority that controls and administers all matters involved in the production and printing of money. However, cryptocurrency, which emerged over a decade ago, is no longer monitored today by any authority in a certain country (DeVries, 2016; Ammous, 2016; Sungit \& Ahmad, 2017; Adam, 2017). Bitcoin is the first cryptocurrency that was developed (2009) and was the first cryptocurrency term that was coined (Albrecht et al., 2019). The term 'cryptocurrency' is used because all transactions and issuance of new units will use the cryptography system that is developed using the blockchain technology. The intended function of the Bitcoin was to be a universal medium of payment that replaces the currency of a certain country (DeVries, 2016). Cryptocurrency is also known as virtual currency or digital currency (Adam, 2017). Scholars and Fatwa institutions all around the world have provided their views related to the use of Bitcoins (Adam, 2017; Oziev \& Yandiev, 2017; Meera, 2018; Abubakar et al., 2018). However, those views cannot be generalised to all digital currencies. Various digital currencies have been created based on the original Bitcoin concept with various functions that has expanded the use of digital currencies (Albrecht et al., 2019). An increase in digital currencies with various functions requires different legislation and syarak guidelines for each of the function developed. Therefore, there are specific characteristics of these digital currencies outlined by scholars as well as certain countries to classify it into several categories of digital currencies monitored by a specific legislation.

\section{The Expansion of Digital Currency}

Before Bitcoin was created, there were several attempts to develop a virtual currency. However, its implementation did not last long because the development of e-business at that time was at the beginning stage and society had greater trust on the credit card compared to the virtual currency system. Besides that, this currency was targeted by syndicates who used it to finance criminal activities (Hooper, 2017). Bitcoin was first used in 2010 
as a medium of exchange at a rate of $\$ 0.0025$ per Bitcoin (Ammous, 2016). The present value per unit of each Bitcoin is around RM32,349 or 7383 USD.

The increase in Bitcoin's value since its inception until today has encouraged various parties to produce digital currency with different characteristics and concepts but based on Bitcoin's original concept (Meera, 2018) known as Alternative Coin or altcoin (Afilipoaie \& Shortis, 2015). Altcoin is a currency based on blockchain technology, which is dissimilar to that used by Bitcoin (Huang et al., 2018). Hence, some Altcoins are not limited to the main function of Bitcoin, which is a medium of payment, rather, there are other functions as well, such as Ripple and Ethereum, that support the building of a smart contract in its blockchain (Inci \& Lagasse, 2019).

Nick Szabo formally defined a smart contract in 1996 as an agreement in digital form, including the protocols used by two parties for implementing the agreement. This definition contains four elements, namely an agreement, its digital form, its implementation by using certain protocols and its execution by the parties involved (Mukhopadhyay, 2018). Each company that wishes to issue a digital asset through a smart contract by Initial Coin Offering (ICO) should prepare a white paper containing detailed information related to financial products, services or instruments that are in offer. ICO is the launching of the online sale of cryptographic assets to fund the development of the blockchain application, selling access to applications built on the blockchain or funding new crypto currencies (Crosser, 2018; Sykes, 2018; Senderowicz et al., 2018; PwC, 2019).

Most Altcoins are created as instruments for speculative investment, whereas Bitcoin was created as a medium of exchange for services or online/offline products (Huang et al., 2018; Inci \& Lagasse, 2019). Some Altcoins have more attractive characteristics compared to Bitcoin, such as a higher level of security and a shorter time for certifying each transaction, which is only a few seconds compared to Bitcoin's minimum time of 10 minutes (Afilipoaie \& Shortis, 2015; Ammous, 2016; Meera, 2018). Nevertheless, Bitcoin is the main benchmark used to measure the value of other Altcoins (Hayes, 2015).

\section{Classification of Digital Currency}

Bitcoin was the first term used to describe a cryptocurrency or digital currency because it was the first digital currency that used the cryptographic system with blockchain technology (Albrecht et al., 2019). The emergence of other digital currencies produced by improving the original Bitcoin concept had various functions and characteristics and was called cryptocurrency (Joo et al., 2019). Now, this term does not only focus on the medium of payment function alone but includes various financial infrastructure and instruments built on the smart contract platform that issues digital currencies with various functions.

Islamic scholars had classified digital currency into several different categories without a specific guide so that it can be uniformly used by all countries. There are three different views about the classification of digital currency according to its functions (Mukhopadhyay, 2018; FINMA, 2018; Howell et al., 2018; Asif, 2018; Sockin \& Xiong, 2018; Wu et al., 2018; Deloitte, 2019; Victor \& Luders, tt). According to several studies (Victor \& Luders (tt), Asif, 2018, Sockin \& Xiong, 2018, Howell et al., 2018, Wu et al., 2018 and Mukhopadhyay, 2018), digital currency can be divided into two categories, which are coins and tokens. Tokens are created through the smart contract platform that is built on the blockchain technology used by other digital currencies, such as ERC-20, that can only be developed using the Ethereum blockchain (Mukhopadhyay, 2018). A token represents an asset or various commodities that can be distributed, such as physical or digital assets, shares, votes, membership, loyalty bonusses and other utilities. Sockin \& Xiong (2018) and Howell et al., (2018) had divided tokens into two categories according to different functions, namely utility and security tokens. Coins represent a digital form currency and is created using a blockchain of its own, such as Bitcoin and Ethereum. Coins have limited function, which is a store of value, compared to tokens that have numerous functions.

Deloitte (2019) and Maxson et al., (2019) had used the term 'crypto asset' because the term 'cryptocurrency' usually refers to a medium of payment, such as Bitcoin. 'Crypto assets' is a much wider term and refers to security tokens or other innovations developed by using the crypto system. Crypto assets comprise three categories, namely payment or exchange tokens, utility tokens and security tokens (Deloitte, 2019; Maxson et al., 2019). Payment tokens are one form of payment for a service or commodity, such as Tether (USDT), which was created based on the US Dollar. Each Tether has a base value of 1 US Dollar in the savings of the Tether Limited company (Tether Limited, tt). Besides Tether, the USD Coin (USDC) was also developed based on the value of the US Dollar (Centre, 2018). The utility token prepares access to the function directly offered by the issuer of the token. For example, the LEO token was created to provide benefits to Bitfinex traders and allows the token holder to obtain better rates and services when using the Bitfinex bursar (iFinex, 2019). The security token prepares asset ownership and the right to use it, dividend pay-outs and the right to vote by the token's owner (Deloitte, 2019). The OmiseGO 
token is one example of a security token. Project OmiseGO is under the auspices of the Omise Holdings Pte Ltd, a company that was formed in 2013. OmiseGO created a platform to manage digital assets, fiat money and other assets such as shares through the Software Development Kit (SDK) wallet (Poon \& OmiseGO Team, 2017).

The Swiss Financial Market Supervisory Authority (FINMA) has divided digital currency into three, which are payment tokens, utility tokens and asset tokens (FINMA, 2018). There is no general classification known as Initial Coin Offerings (ICO) in Switzerland or any other country; hence, FINMA had categorised digital currency according to its economic function based on a certain digital currency's ICO. Payment tokens were created to be used now or in the future, with the aim of paying for goods or services, substituting the function of money as well as an exchange of value. Utility token is a token that allows access for an application or service on the blockchain infrastructure. The asset token represents assets such as debts or equity from the producer of the token. An example of an asset token, such as ownership or profit in a company, is similar to equity, bonds or derivatives in a traditional system. Moreover, each token is not limited to only one function. According to FINMA (2018) and Deloitte (2019), a token that combines the function of the utility or security tokens with the medium of payment is known as a hybrid token. Tokens that provide access to the sale and purchase of physical assets on the blockchain technology are also included in the asset token category. All asset tokens and some utility tokens that are created for investment, either as the main aim or supplementary aim to the function of the utility token, is also presumed to be a security token.

Countries that place digital currency under the security legislation, such as the US and Switzerland, usually only refer to digital tokens that have security characteristics that are similar to traditional financial instruments. The U.S. Securities and Exchange Commission (SEC) is the agency in charge of monitoring Initial Coin Offerings (ICO) in the U.S. The legal approach taken by SEC stipulates that the Howey Test should be applied in every Initial Coin Offering (ICO) in order to classify the cryptocurrency (Senderowicz et al., 2018). ICO is the online sale of cryptographic assets aimed at funding the blockchain application development project, selling access to applications built on a blockchain or seeking funds for a new cryptocurrency. The Howey Test is intended to determine whether a financial instrument or digital token can be categorised as a security token. Thus, if a digital currency fulfils the Howey Test, then it is categorised as a security token. The Howey Test contains 4 questions that involve financial investment, anticipation of profits, investment in the same industry and the prediction of profits by other parties (Crosser, 2018; Sykes, 2018; Senderowicz et al., 2018; PwC, 2019).

The three views in focus have clear similarities and only one difference, namely classifying a part of the utility token as a security token by FINMA, which is a more general view (Victor \& Luders, tt; Mukhopadhyay, 2018; Asif 2018; Wu et al., 2018; Mukhopadhyay, 2018) compared to the other two. They had divided it only into two categories, which are coins and tokens, without classifying the specific function in the token. Sockin \& Xiong (2018) and Howell et al., (2018) had divided tokens into two categories according to different functions, namely utility and security tokens. There are similarities in the classification of digital currency between FINMA as well as Deloitte, 2019 and Maxson et al., 2019 because FINMA also considers the asset token to be a security token. Therefore, all legislation and guidelines used in securities should be applied in the use of asset tokens (FINMA, 2018).

Classifying digital currency according to Malaysian law is different compared to the three views mentioned earlier. The main classification of cryptocurrency is divided into two categories, namely digital currency and tokens. Malaysia has established certain methods for digital currency and tokens before enforcing legislation on securities. The Attorney General's Department, as published in a Federal Government Gazette under the Capital Markets and Services (Prescription of Securities) (Digital Currency and Digital Tokens) Order 2019, had established a specific definition for classifying cryptocurrency or also known as digital assets (SC, 2019b). In this Order, digital currency means a representation of digital value recorded in a distributed digital ledger, either it is cryptographically secured or otherwise, which functions as a medium of exchange and can be exchanged with any money, including the crediting or debiting of an account. Digital tokens refer to a digital representation that is recorded in a distributed digital ledger, either it is cryptographically secured or otherwise.

Digital currency is presumed to be a security when fulfilling three situations. First, when it is traded in a place or facility where the offer to sell, purchase or exchange digital currency is usually made or accepted. Second, when someone hopes for returns in any form from the trading, exchanging or redeeming of digital currency or an increase in the value of the digital currency. Third, when the digital currency is not issued or guaranteed by any government body or central bank as determined by the Commission (SC, 2019b).

A digital token represents a person's right or interest in any calculation or consideration made towards or has an effect when assisting the owner of the digital token. The token represents various types of tangible goods that 
can be transferred (ownership and possession), such as digital or physical assets, shares, voting rights, loyalty and membership bonuses as well as other utilities (Blockchain, $\mathrm{tt}$ ). The digital token is presumed to be a security if it satisfies six conditions, such as when the investor or buyer of the digital token receives the digital token as an exchange for a requital. Second is the returns or contributions to the investor as well as the income or accumulated returns. Third is income or calculated returns generated from acquisitions or procurements, tenures and management or disposal of property, assets or business activities. Fourth, investors or holders of digital assets hope for returns in any form from trading, exchanges or redeeming of digital tokens or increase in the value of the digital token. Fifth, the digital asset holder does not have day-to-day control over the management of the property, asset or businesses and sixth, the digital token is not issued or guaranteed by any government body or central bank as determined by the Commission (SC, 2019b).

Digital currency and tokens, which are presumed to be securities, related to current financial instruments are enforced under security laws. Digital currency and tokens that are classified as security and offered or traded on or through the market are not shares in a debenture, a corporate body or an unincorporated body. Both are units in a unit trust scheme or investment scheme (SC, 2019b). Rapid development in technology cannot be curtailed by both these entities. Although some views said that cryptocurrency would only last for a few years after its first creation, which was Bitcoin, the reality now clearly contradicts that view. Hence, Malaysia had taken the approach of formulating guidelines and legislation related to digital assets, which includes digital currency and tokens. There were several changes to the guidelines issued by the Security Commission of Malaysia (SC), which is the body responsible for monitoring and issuing guidelines related to digital currency. The guidelines are consistent with matters outlined by other bodies in Malaysia, such as Bank Negara Malaysia (BNM).

On 19 January 2018, the SC and BNM had issued statements that any activity involving Initial Coin Offerings (ICO) carried out without proper approval is an offence. The offer of digital tokens to be changed into digital currency or any form of payment and incidental activity calls for monitoring under the security law, which is subject to legislation administered by SC and BNM. Hence, all activities that are monitored, such as obtaining funds as well as managing funds and transactions related to capital market products without the permission and approval from the SC cannot be carried out by any individual or company (BNM, 2018).

On 6 December 2018, SC and BNM had issued a subsequent statement saying that the SC will monitor the issuing of digital assets by Initial Coin Offerings (ICO) and the trading of digital assets in the digital asset bursar in Malaysia. The issuing of ICO and the digital asset bursar involved in issuing or dealing in the sales and purchase of digital assets should adhere to the legislation and BNM regulations related to payment and currency dealings. Besides that, issuing of ICO and the digital asset bursar are subject to the Prevention of Money Laundering Policy and Counter Financing of Terrorism (CFT) in Digital Currency. Hence, BNM delivered a reminder that digital assets are legally uncertified in Malaysia and the public is advised to be careful in evaluating related risks when dealing with digital assets (BNM, 2018).

In March 2019, SC had issued a consultation paper related to the framework for collecting funds by offering digital tokens in order to obtain feedback from any party (SC, 2019a). Based on the response received, there was encouraging support from the industry regarding SC's suggestion to use experts running these platforms to evaluate applications for issuing digital tokens to collect funds. Then on 15 January 2020, the SC issued a Digital Asset Guideline that outlined a framework for collecting funds by issuing digital tokens in Malaysia. The guideline stipulated requirements for all digital token offers implemented through a platform manager registered with the SC. The offer was known as the Initial Exchange Offering (IEO).

Every company that intends to issue a digital token for fund collection must go through a bursar that authorised by the SC and it cannot be sold by the company itself. The platform manager will evaluate the business of the issuer, the qualifications and compatibility of the issuer's board of directors as well as understand the characteristics of the digital token to be issued. Prospective issuers are required to fulfil the governance and capital needs that would qualify them to collect funds by offering digital tokens. The issuer is required to show that the suggested project or business provides innovative solutions or suggests the best digital value for Malaysia. These guidelines would be enforced in the second half of 2020 in order to enable issuers, platform managers and investors to familiarise themselves with the guidelines (SC, 2020).

Although the IEO guidelines were issued by the SC in order to replace the ICO; however, digital assets cannot be certified in Malaysia (SC, 2020). Therefore, the guidelines prepared by the Malaysian government is a step taken to protect investors from fraudulent schemes that promise various profits. Although the projects stated in the proposal have been sent to the platform managers, it might not be entirely successful; hence, at least the projects 
will be implemented according to what has been stated under the supervision of the authorities, contrary to the fraudulent schemes that collect funds without any effort (Azzuhri, 2020).

\section{Analysis}

Classifying different digital currencies needs syarak guidelines drawn according to different characteristics of each digital currency. All payment and exchange transactions involving the coins, payment tokens and digital currency categories must be carried out fairly and without coercion. The token issuer who prepares access to services and infrastructure developed by the smart contract platform must explain the operations carried out in order to avoid any confusion among investors and clients. The products and services offered must be Syariah compliant and free from elements involving gambling or criminal activities. Countries around the world have different views and acceptance about the use of digital currency, either according to the inherent function of the digital currency or generalisations. Moreover, the legislation in force differs between each country. The use of cryptocurrency, either in the form of digital currency or assets, is not recognised or certified in Malaysia. BNM has placed digital currency under the Anti-Money Laundering (AML) and Counter Financing of Terrorism (CFT) Acts. These Acts will protect consumers from getting involved in transactions related to criminal activities and money laundering (BNM, 2018).

However, the bursar enforces legislation that involves the selling and purchasing of digital currencies. There are three bursars for exchanging digital currencies approved by the Malaysian government under the supervision of the SC (SC, tt). The SC does not permit any Initial Coin Offerings (ICO) or Security Coin Offerings (SCO) to be carried out in Malaysia. In January 2020, the SC had issued guidelines that permit Initial Exchange Offerings (IEO) to be carried out in Malaysia. This shows the readiness of Malaysia to develop blockchain technology. Nevertheless, digital assets are still not legally endorsed in Malaysia and the SC will only supervise sales and purchase transactions at a certain bursar that satisfies the stipulated qualifications (SC, 2020). Hence, any party that offers and accepts digital assets when carrying out any business dealings is doing so at one's own risk. According to a report in the Berita Harian (2017), consumers of digital currency are not protected under dispute resolution regulations if there are disputes or losses. Although BNM has issued the Money Laundering Prevention Policy and Countering Terrorism Financing for Digital Currency, this does not mean that BNM allows, endorses, licenses or supports any entity involved in digital currency exchange services. A consumer who falls victim to cryptocurrency or digital asset cheating cases is presumed not to experience any losses because the status of the digital asset is not legally endorsed in Malaysia (Shariffuddin, 2020). Table 1 shows the type and category of digital currency based on previous studies.

Table 1. Classification of Digital Currency According to Function

\begin{tabular}{|c|c|c|c|c|c|c|c|c|}
\hline \multirow{2}{*}{$\begin{array}{l}\text { Type of Digital } \\
\text { Currency }\end{array}$} & \multicolumn{8}{|c|}{ Category of Digital Currency } \\
\hline & Asset & Coin & Currency & Token & $\begin{array}{c}\text { Payment } \\
\text { Token }\end{array}$ & $\begin{array}{c}\text { Utility } \\
\text { Token }\end{array}$ & $\begin{array}{c}\text { Asset } \\
\text { Token }\end{array}$ & $\begin{array}{c}\text { Security } \\
\text { Token }\end{array}$ \\
\hline Bitcoin & $\mathrm{x}$ & $\mathrm{x}$ & $\mathrm{x}$ & & & & & \\
\hline Ethereum & $\mathrm{x}$ & $\mathrm{x}$ & $\mathrm{x}$ & & & & & \\
\hline ERC-20 & $\mathrm{x}$ & & & $\mathrm{x}$ & & & & \\
\hline Tether & $\mathrm{x}$ & & $\mathrm{x}$ & & $\mathrm{x}$ & & & \\
\hline USD Coin & $\mathrm{x}$ & & $\mathrm{x}$ & & $\mathrm{x}$ & & & \\
\hline LEO & $\mathrm{x}$ & & & & & $\mathrm{x}$ & & \\
\hline OmiseGO & $\mathrm{x}$ & & & & & & $\mathrm{x}$ & $\mathrm{x}$ \\
\hline
\end{tabular}

Source: Victor \& Luders, tt; Asif, 2018; Sockin \& Xiong, 2018; Howell et al.,2018; Wu et al., 2018; Mukhopadhyay, 2018; FINMA, 2018; Deloitte, 2019; Maxson et al., 2019; Tether Limited, tt; Centre; 2018; Poon \& OmiseGO Team, 2017; iFinex, 2019.

Table 1 illustrates the classification of digital currency according to its functions developed for each digital currency. The term 'cryptocurrency' is used because the term usually refers to its function as a medium of payment, such as Bitcoin (Deloitte 2019; Maxson et al., 2019). Malaysia also uses the term 'digital asset', which includes currency and tokens (SC, 2019b). Currency is a medium of exchange and is exchangeable with any type of money, including the crediting or debiting of accounts (SC, 2019b). There are various views related to the classification of digital currency (Victor \& Luders, tt; Asif, 2018; Sockin \& Xiong, 2018; Howell et al.,2018; Wu et al., 2018; Mukhopadhyay, 2018; FINMA, 2018; Deloitte, 2019; Maxson et al., 2019). There are two types of digital currency, namely coins and tokens (Victor \& Luders, tt; Asif, 2018; Sockin \& Xiong, 2018; Howell et al.,2018; Wu et al., 2018; Mukhopadhyay, 2018). Coins function as a medium of payment for goods or services as well as a store of value that is built on its blockchain, such as Bitcoin and Ethereum. Moreover, tokens are built with numerous 
functions, besides the medium of payment function, on other digital currency blockchains, such as ERC20, which is built on the Ethereum blockchain (Mukhopadhyay, 2018).

Tokens can be divided into two categories, namely utility and security tokens (Sockin \& Xiong, 2018; Howell et al., 2018). However, one view divides digital currency into three categories, such as payment or exchange token, utility token and security or asset token (FINMA, 2018; Deloitte, 2019; Maxson et al., 2019). Tether and USD Coins are examples of payment tokens, which are developed to replace fiat money in the form of cryptocurrency. Each token is based on the value of 1 USD (Tether Limited, tt; Centre; 2018). The utility token prepares access to the function directly offered by the issuer to the token's owner. For example, the LEO token was created to provide benefits to Bitfinex traders (iFinex, 2019). The security or asset token provides asset ownership and the right to use it as well as dividends and the right to vote by token owners (Deloitte, 2019). The OmiseGO Token is one example of a security token. The OmiseGO creates a platform for managing digital assets, fiat money and other assets, such as shares, through the Software Development Kit (SDK) wallet (Poon \& OmiseGO Team, 2017).

The digital asset or crypto asset has a much wider meaning and includes all functions found in the digital currency. According to the characteristics and functions that have been developed, Bitcoin and Ethereum are categorised as coins and currency. ERC20 is a standard for tokens, which can be developed using the Ethereum blockchain, and is categorised as a token, in general. Tether and USD Coin are categorised as currency and payment tokens. LEO, which was developed to provide benefits for the owner, is categorised as a utility token. OmiseGO is categorised as a security and asset token by offering a platform for financial management.

\section{Conclusion}

Numerous countries have beefed up steps to ensure that they are not left behind in the technological race, mainly in terms of national procurement and economic activities. Malaysia has issued guidelines for digital currency transactions that are permitted in Malaysia. The categorisation of digital assets into digital currency and digital tokens as well as establishing a specific method for classifying both as a security token will facilitate enforcement of the developed technology. The development of legislation related to digital currency since 2018 shows the readiness of Malaysia to benefit from blockchain technology that has been adopted by other countries. Hence, its implementation must be consistent with the demands of Islam. Islamic scholars' views about determining the religious decree concerning digital currency based on the Bitcoin concept cannot be applied to other digital currencies that are based on different concepts. Therefore, Malaysia needs to have a specific guideline for this technology, including guidelines for the companies or investors involved and products that are offered so that it can be implemented without transgressing syariat Islam. This is important as it ensures that Malaysia is always ahead of other developed countries. Thus, if the whole world uses blockchain technology and digital currency as a medium for economic activities, then, countries that do not use that technology would face difficulties when collaborating or cooperating with other countries.

\section{Acknowledgement}

This research was funded by the Ministry of Higher Education Malaysia under the research grant fund, namely the Fundamental Research Grant Scheme (FRGS) FRGS/1/2018/SS08/UNISZA/02/1 entitled 'A Guideline for Syarak in Relation to Digital Currency Transactions'.

\section{References}

1. Abubakar, Y.S., Ogunbado, A.F., \& Saidi, M.A. (2018). Bitcoin and its legality from Shariah point of view. SEISENSE Journal of Management, 1(4), 13-21.

2. Adam, M.F. (2017). Bitcoin: shariah compliant. Amanah Finance Consultancy, 1-54.

3. Afilipoaie, A., \& Shortis, P. (2015). From Dealer to Doorstep-How Drugs Are Sold on the Dark Net. GDPO Situation Analysis. Swansea University: Global Drugs Policy Observatory.

4. Albrecht, C., Duffin, K.M., Hawkins, S., \& Rocha, V.M.M. (2019). The use of cryptocurrencies in the money laundering process. Journal of Money Laundering Control. 22(2), 210-216.

5. Ammous, S. (2016). Can cryptocurrencies fulfil the functions of money? Columbia University, Center on Capitalism and Society, Working Paper No. 92.

6. Asif, S. (2018). The halal and haram aspect of cryptocurrencies in Islam. Journal of Islamic Banking and Finance, 35(2), 92-102.

7. Azzuhri, Saaidal Razalli. (2020). Ethereum's operational concept and its development challenges in Malaysia. Faculty of Computer Science and Information Technology University of Malaya. Interviews.

8. Berita Harian. (2017). Digital currency is not recognized. 
9. https://www.bharian.com.my/bisnes/lain-lain/2017/12/363899/mata-wang-digital-tidak-diiktiraf.

10. Blockchain. $\quad(\mathrm{tt})$. $\quad$ The State of $\quad$ Stablecoins. https://www.blockchain.com/ru/static/pdf/StablecoinsReportFinal.pdf.

11. BNM. (2018). Anti-Money Laundering and Counter Financing of Terrorism (AML/CFT) - Digital Currencies (Sector 6). https://www.bnm.gov.my/index.php?ch=57\&pg=538\&ac=680\&bb=file.

12. Centre. (2018). USD Coin Whitepaper. https://www.centre.io/pdfs/centre-whitepaper.pdf.

13. Coinmarketcap.com. (2019). All cryptocurrencies by Market Capitalization.

14. https://coinmarketcap.com/all/views/all/.

15. Crosser, N. (2018). Initial coin offerings as investment contracts: Are blockchain utility tokens securities. University of Kansas Law Review 67, 379.

16. Deloitte. (2019). Are Token Assets The Securities of Tomorrow?

17. DeVries, P.D. (2016). An analysis of cryptocurrency, bitcoin, and the future. International Journal of Business Management and Commerce, 1(2), 1-9.

18. FINMA, S.F.M.S.A. (2018). Guidelines for enquiries regarding the regulatory framework for initial coin offerings (ICOs).

19. Hayes, A. (2015). What Factors Give Cryptocurrencies Their Value: An Empirical Analysis. 1-6.

20. Hooper, K. (2017), Bitcoin: Digital Currency or Digital Tulip?. Advisor Perspective. 1-4.

21. Howell., S.T, Niessner., M, \& Yermack, D. (2018). Initial Coin Offerings: Financing Growth with Cryptocurrency Token Sales.

22. Huang, D.Y., Levchenko, K., \& Snoeren, A.C. (2018). Estimating Profitability of Alternative Cryptocurrencies (Short Paper). In International Conference on Financial Cryptography and Data Security , 409-419.

23. IFinex. (2019). LEO Whitepaper. https://www.bitfinex.com/wp-2019-05.pdf.

24. Inci, A.C., \& Lagasse, R. (2019). Cryptocurrencies: applications and investment opportunities. Journal of Capital Markets Studies, 3(2).

25. Joo, M.H., Nishikawa, Y., \& Dandapani, K. (2019). Cryptocurrency, a successful application of blockchain technology. Managerial Finance.

26. Maxson, S. Davis, S. and Moulton, R. (2019). UK Cryptoassets Taskforce publishes its final report. Journal of Investment Compliance. 20(2), 28-33.

27. Meera, A.K.M. (2018). Cryptocurrencies from islamic perspectives: The case of bitcoin. Buletin Ekonomi Moneter Dan Perbankan, 20(4), 475-492.

28. Mohamad, Zulkifli (2018). Bayan Linnas 153rd Series: Bitcoin Currency Usage Law. https://muftiwp.gov.my/ms/artikel/bayan-linnas/2773-bayan-linnas-153-hukum-penggunaan-matawang-bitcoin.

29. Mukhopadhyay, M. (2018). Ethereum Smart Contract Development: Build blockchain-based decentralized applications using solidity. Packt Publishing Ltd.

30. Oziev, G., \& Yandiev, M. (2017). Cryptocurrency from Shari'ah perspective. Available at SSRN 3101981 .

31. Poon, J., \& Team, O. (2017). OmiseGo Decentralized Exchange and Payments Platform. White paper. https://whitepaperdatabase. com/omisego-omg-whitepaper.

32. PwC. (2019). Security Token Offering: what, where and how.

33. SC. (tt). List of Registered Recognized Market Operators. https://www.sc.com.my/development/digital/list-of-registered-recognized-market-operators.

34. SC. (2017). Media Statement on Initial Coin Offerings. https://www.sc.com.my/resources/mediareleases-and-announcements/media-statement-on-initial-coin-offerings.

35. SC. (2019a). Proposed Regulatory Framework for the Issuance of Digital Assets Through Initial Coin Offerings (ICOs).

36. https://www.sc.com.my/api/documentms/download.ashx?id=d3067bf4-ba39-4bce-8dc4-1b660ff37977.

37. SC. (2019b). Capital Markets and Services (Prescriptions of Securities) (Digital Currency and Digital Token).

38. https://www.sc.com.my/api/documentms/download.ashx ?id=8c8bc467-c750-466e-9a8698c12fec4a77.

39. SC. (2020) Digital Assets Guidelines.

40. https://www.sc.com.my/api/documentms/download.ashx?id=dabaa83c-c2e8-40c3-9d8f-1ce3cabe598a.

41. Senderowicz., J.I, Grafton., K.S, Spangler., T, Brown., K.D., \& Schaffer., A.J. (2018). SEC focuses on initial coin offerings: tokens may be securities under federal securities laws. Journal of Investment Compliance. 19(1), 10- 14.

42. Sockin., M, \& Xiong., W. (2018). A Model of Cryptocurrencies.

43. Shariffuddin, Aliff. (2020). Focus Group Discussion on Digital Currency. Kulliyah of Economics and Management Sciences, International Islamic University Malaysia. 
44. Sungit, F., \& Ahmad, K.A. (2017). Shariah Issues Against Bitcoin: An Early Highlight. 3rd Muzakarah Fiqh \& International Fiqh Conference (3rd MFIFC) Shah Alam.

45. Sykes, J.B. (2018). Securities Regulation and Initial Coin Offerings: A Legal Primer.

46. Tether Limited. (tt). Tether Whitepaper.

47. https://tether.to/wp-content/uploads/2016/06/TetherWhitePaper.pdf.

48. Victor., F., \& Luders, B.K. tt Measuring Ethereum-based ERC20 Token Networks.

49. Wu, K., Wheatley, S., \& Sornette, D. (2018). Classification of cryptocurrency coins and tokens by the dynamics of their market capitalizations. Royal Society open science, 5(9), 180381. 